\title{
Significado psicológico del concepto investigación en investigadores ${ }^{*}$
}

\section{Psychological meaning of the concept of "research" in researchers}

\author{
Dary Lucía Nieto Súa** \\ Nubia Yaneth Gómez Velasco \\ Stephania Eslava \\ Universidad Pedagógica y Tecnológica \\ de Colombia
}

Recibido: 8 de octubre de 2015 Revisado: 30 de noviembre de 2015 Aceptado: 22 de diciembre de 2015

\section{Resumen}

Esta investigación se planteó como objetivo establecer el significado psicológico de la "actividad de investigar", para un grupo de profesionales de las Ciencias Sociales. Se usó la técnica de Redes Semánticas Naturales, versión definida por Valdez (2004), que hace énfasis en el análisis del significado psicológico construido a partir de las vivencias. El instrumento fue aplicado a 46 asistentes de un evento académico en el área de Ciencias Sociales, elegidos mediante un muestreo no probabilístico intencional. Los resultados muestran un total de 220 palabras diferentes asociadas al término investigación (valor J). Los dos términos de mayor peso semántico fueron indagación (106) y búsqueda (101), en contraste con las palabras análisis y disciplina, cuyo peso semántico fue 33. A través de una prueba de independencia entre las variables sociodemográficas y el grupo SAM, se evidenció que no existe relación significativa con el género y la edad.

Palabras clave: redes Semánticas Naturales, investigación, significado psicológico, Ciencias Sociales. 


\section{Abstract}

This research had the objective of establishing the psychological meaning associated with the "research activity" for Social Science Professionals. We used the Natural Semantic Network technique (Valdez, 2004), which emphasizes the analysis of the psychological meaning constructed from the experiences. The instrument was applied to 46 attendees of an academic event in the area of Social Sciences, in an intentional non-probabilistic sampling. The results show a total of 220 different words associated with the term "Research" ( $\mathrm{J}$ value). The two terms of highest semantic weight were: Inquiry (106), Search (101), in contrast with the words Analysis and Discipline with semantic weight of 33. Through a test of independence between sociodemographic variables and the SAM group, no significant relationship with gender and age emerged.

Keywords: Natural Semantic Networks, research, psychological meaning, Social Sciences.

\section{Introducción}

La búsqueda de conocimiento presente en toda la historia del ser humano, ha influido de manera significativa en el proceso de desarrollo histórico de la humanidad, desde el conocimiento sistemático y riguroso, que se ha denominado científico, hasta aquel que se obtiene en la cotidianidad. Este conocimiento recogido en teorías, conceptos, instrumentos y técnicas explicativas de los fenómenos de la naturaleza, es el resultado de un procedimiento.

La investigación ha sido entendida como el proceso de construir conocimiento a partir del análisis sistemático, riguroso y formal, como fuente del verdadero conocimiento; esta forma de adquirir conocimiento, según Ortiz (2012), fue desarrollada en los inicios del siglo XX y generó un cambio en las prioridades e intereses de grupos sociales. Hacer investigación va más allá del uso de una serie de técnicas e instrumentos, requiere de una postura epistemológica y metodológica del investigador, pues no tenerlas, implica que el proceso se quede en una simple recolección de información, sin la posibilidad de socializar para que aporte al desarrollo de la humanidad.

Diferentes posturas epistemológicas de historiadores como Hergenhahn (2008), ponen de manifiesto la dialéctica entre el conocimiento producto de un procedimiento cuantitativo y uno cualitativo; evidenciando, según el historiador, cierta preferencia por la investigación cuantitativa, por su cercanía con las llamadas ciencias duras, al validar los conocimientos desde análisis matemáticos. En los inicios del siglo XX (Leahey, 1998), se da el desarrollo de las ciencias sociales, lo que permitió una mayor comprensión del ser humano en sus dimensiones éticas, estéticas, axiológicas y ontológicas; dimensiones difíciles de explicar desde un enfoque cuantitativo, lo que favoreció el desarrollo de los enfoques cualitativos. La situación descrita plantea que realizar investigación cuantitativa o cualitativa sugiere un paradigma que pueden condicionar los problemas por resolver, los fenómenos por explicar, los procedimientos e incluso la interpretación o análisis de la información obtenida.

\section{Reflexión sobre el concepto de investigar}

Mucho se ha escrito sobre los diferentes tipos de investigación entre las disciplinas, así como sobre las terminologías específicas; el abordaje de las múltiples posturas y enfoques en investigación hace que, en ocasiones, genere confusión más que claridad respecto a los procesos adecuados para la investigación. Sin embargo, independientemente de la multiplicidad de criterios para desarrollar investigación, un aspecto que no escapa a ninguno de estos es el método. Sin un método 
definido no habría posibilidad de desarrollo de la ciencia, sin desconocer que cualquier metodología elegida tenga debilidades y limitaciones. Es así, que reflexiones y debates sobre los métodos de investigación, en búsqueda de resultados más confiables, han permitido un acercamiento cada vez mayor a los paradigmas cualitativos, y con ellos una significativa contribución a la comprensión integral del ser humano.

Comprender al ser humano de manera integral es aceptar su complejidad, en el mundo en el que hoy nos desenvolvemos se plantea la necesidad de investigar desde diferentes disciplinas, para obtener conocimiento de los entornos en los que se encuentra inmerso cada individuo. A través de la historia no se ha modificado el interés por entender al hombre y su relación con el universo, han cambiado las metodologías: de ser explicaciones filosóficas a utilizar procedimientos validados reconocidos como investigación científica.

¿Qué o quién valida hoy en día la investigación como proceso que aporta verdades sobre el conocimiento del ser humano y su entorno? Son muchos los que hacen investigación y algunos se interesan por su ontología.

Pero, ¿qué es investigar? Hay un buen número de definiciones de investigar, algunas de ellas elegidas para soportar el objetivo de este artículo, contemplan la reflexión como elemento fundamental:

La investigación permite cuestionar, reflexionar y actuar sobre el acontecer histórico y social en la medida que favorece un criterio propio, fundamentado científicamente; criterio que beneficia a poblaciones atendidas, la creación novedosa de estrategias y métodos de intervención, el aumento de la calidad de la investigación, el rendimiento y respeto de la imagen profesional (Vargas, 2009, p. 158).

Autores como Ander-Egg (1992) afirman que la investigación es:

Un procedimiento reflexivo, sistemático, controlado y crítico que tiene por finalidad descubrir o interpretar los hechos y fenómenos, relaciones y leyes de un determinado ámbito de la realidad [...] una búsqueda de hechos, un camino para conocer la realidad, un procedimiento para conocer verdades parciales 0 , mejor, para descubrir no falsedades parciales (p. 57).

Para otros investigadores como Cívicos (2007), consideran que la investigación requiere de un procedimiento sistemático, lo que la convierte en una actividad densa y compleja, más aún cuando una investigación se realiza sobre un tema poco investigado. Este mismo autor plantea que más allá del modelo cuantitativo y cualitativo, se hace investigación básica, que se ocupa del objeto de estudio sin considerar una aplicación inmediata, y cuyos resultados y descubrimientos pueden originar nuevos productos y avances científicos (Cívicos y Hernández, 2007), e investigación aplicada, que es aquella que utiliza los conocimientos en la práctica. Por su parte Padrón (2006) y Martínez (2004) enfatizan que los conocimientos son para aplicarlos en provecho de la sociedad en general, además de ofrecer nuevos conocimientos que enriquecen la disciplina desde la cual se investiga.

Se puede decir, entonces, que describir, interpretar y analizar fenómenos sociales y humanos en contextos reales es la finalidad de la investigación en Ciencias Sociales, ya que estas pueden y deben reivindicar la pertinencia social, científica y humana, invitar a reflexionar y poner en debate todos los temas concernientes a la elaboración de posibles alternativas económicas y sociales que la sociedad requiere.

En el contexto académico la investigación se ha convertido en parte fundamental del proceso formativo, en evidencia de estar preparado para recibir un título profesional; por ello, y como lo plantea Cordero (2009), la investigación requiere rigurosidad y excelencia que favorezcan la toma de conciencia y el compromiso del investigador con la realidad, de modo que se logren tanto soluciones a los fenómenos estudiados como cambios epistemológicos y paradigmáticos en los investigadores. Es decir, y como lo plantea Best (1982), el término ciencia ha llegado a significar no solo un método, o un conjunto de contenidos, sino 
una actitud. Se plantea entonces que investigar requiere de una disposición anímica ante lo que se va a estudiar.

Por ello, la motivación de quien investiga es un factor fundamental en este proceso; la mayoría de investigaciones se realizan con el propósito de obtener un beneficio, ya sea para el investigador o para el contexto de la investigación. García y Doménech (1997) plantean que la motivación puede ser extrínseca o intrínseca; la extrínseca es definida como aquella que procede de fuera, como cooperar con las necesidades que se presentan en la sociedad o con los requerimientos de las diferentes disciplinas; y la intrínseca, como aquella que surge del propio sujeto, que está bajo su control y tiene capacidad para autorreforzarse, como el reconocimiento personal en el ámbito científico.

En el aspecto motivacional influyen diferentes factores, como la falta de recursos y de apoyo de las instituciones a las que pertenecen los investigadores y la disponibilidad de recursos bibliográficos y de apoyo técnico dentro de la institución en que se desarrolla la investigación, tema de análisis de Aceituno, Lanz, Lanz y Lander (1998), quienes encontraron una clara relación entre la motivación para adelantar un tema de investigación y lo encontrado en ella.

\section{Memoria y redes semánticas}

Conocer las motivaciones de los investigadores permite prever los alcances y limitaciones del estudio que se adelanta, ya que, como señalan Tapia, Huerta y Ruiz (2010), se establecen metas específicas como aprender, encontrar la solución para ayudar a otras personas y adquirir desarrollo intelectual, entre otras. Así, la motivación se convierte en parte del sistema simbólico de los investigadores, en un elemento primordial en la construcción del significado de la actividad que realizan.

Desde una perspectiva psicológica es necesario tomar en cuenta el mundo de significados ligado a la experiencia de cada sujeto, así como su edad, nivel de maduración; en este sentido se explica que la conducta de las personas surge de los procesos de retroalimentación a nivel mental de la información, que se elabora dentro de un contexto.

A partir de este fundamento, el desarrollo de la técnica de Redes Naturales se considera un método confiable para comprender la forma en que se asocia a un significado individual y la estructura de la memoria que lo evoca. Para Salas-Menotti (2008) la técnica de Redes Semánticas permite "obtener una idea clara de los aspectos sobresalientes, dirección, profundidad e intensidad generales de los constructos que se están investigando, así como aspectos culturales y conductuales" (p. 332).

La técnica de Redes Semánticas es un procedimiento de obtención de información relevante dentro del significado psicológico de los sujetos. Dentro de las bases teóricas de la técnica está Osgood, Suci y Tannenbaum (1957), quienes desarrollaron un instrumento de evaluación psicológica fundamentado en que un concepto adquiere significado, cuando un signo (palabra) puede provocar la respuesta que está asociada al objeto que representa; Moscovici (1984), quien define las representaciones colectivas como "un mecanismo explicativo de ideas o creencias denominadas fenómenos que necesitan ser descritos y explicados; tales fenómenos se relacionan con una manera particular de entender y comunicar, manera que crea la realidad y el sentido común" (pp. 3-63).

A partir de los estudios anteriormente citados y de autores como Figueroa (1980) y Valdez (2004); el concepto significado psicológico será entendido para este estudio como el conjunto de palabras elegidas por un sujeto a través del proceso cognitivo de reconstrucción de memoria de una palabra estimulo, que además es definido con el nivel de importancia o relevancia del concepto a analizar y la interacción con sus emociones. Por lo tanto, y según Valdez (2004), el significado psicológico “es en sí mismo una unidad natural de estimulación en la producción de las conductas, sean individuales o sociales" (p. 17).

Para la construcción del significado, la memoria juega un papel importante, al permitir recopilar información necesaria en determinado momento. Colom y Flores-Mendoza (2001) plantean que el 
proceso de la memoria está compuesto por varias etapas: la codificación, que se centra en la percepción de la información; el almacenamiento, que consiste en el mantenimiento de esa información; y la recuperación, que se basa en el recuerdo o evocación de la información previamente almacenada.

La técnica de Redes Semánticas implica además el concepto de Memoria Semántica, que para Vera, Pimentel y Batista (2005), se plantea como la construcción de conceptos a partir de los que previamente se tienen, y que posteriormente, desde el significado, permiten establecer relaciones que expliquen la realidad a la que se desea referir, la organización del conocimiento a través de palabras y símbolos verbales interrelacionados a manera de nodos conceptuales que dan origen a conceptos más generales, y es esta interacción la que da origen a las redes semánticas.

Así, la memoria semántica es aquella interpretación interna que hace el individuo para construir la realidad, y a través de la cual las experiencias, las predicciones y causalidades se conectan dentro de combinaciones nuevas. Este proceso dio pie a la Técnica de Redes Semánticas, iniciada por Figueroa (1980) y desarrollada por Valdez (2004), quien la define como:

Técnica que permite recolectar la información perteneciente a nivel memorístico y usada en la construcción de conceptos, con el objetivo de obtener el significado psicológico del concepto a partir de los núcleos de red, que actúan como unidad fundamental de la organización cognoscitiva, compuesta por elementos afectivos y de conocimiento (p. 82).

Por su parte, Rips, Shoben y Smith (1973), introducen el concepto de distancia semántica, refiriéndose a la proximidad existente entre los conceptos según los agrupamientos de rasgos semánticos dados por los sujetos.

En síntesis, hablar de Redes Semánticas es hablar sobre teoría de la estructura y el procesamiento de la memoria; esto significa que: a) cuantas más propiedades en común tienen dos conceptos, más próximamente relacionados se hallan; b) los nom- bres de los conceptos son guardados en una red lexical organizados por similitud fonológica y ortográfica, y c) la persona puede controlar cuándo produce una relación por proximidad, similitud o por ambas.

Dada la importancia de investigar para el profesional hoy en día, y los cuestionamientos que se suscitan sobre qué es "investigación científica", la validez de sus productos y la relación que existe con la motivación de quienes realizan dicho proceso, cobra importancia conocer cuál es el significado psicológico del concepto investigación en profesionales de las Ciencias Sociales, y determinar si los significados difieren significativamente dependiendo del sexo y la edad.

\section{Metodología}

La investigación fue de tipo exploratorio; según Ávila, (2006), este tipo es pertinente en estudios en los que se dispone de muy poca información, lo que permite documentar el tema objeto de estudio. Para efectos de este trabajo se buscó un acercamiento al significado psicológico del concepto investigación con la Técnica de Redes Semánticas, establecida por Valdez (2004). El enfoque de esta investigación fue mixto, cualitativo-cuantitativo.

Desde la teoría expuesta por Valdez (2004), la Técnica de Redes Semánticas Naturales requiere establecer valores referenciales para el análisis, entre ellos:

- Valor J: representa el total de palabras definidoras que generan los sujetos, y determina la riqueza semántica de la red conceptual.

- Valor M: representa un indicador del peso semántico obtenido para cada una de las palabras definidoras, dado por el valor jerárquico asignado.

- Grupo SAM: corresponde al grupo de n-palabras que obtienen los mayores valores $M$ totales. Para este estudio se estableció $n=15$ (Según Valdez 2004).

- Valor FMG: indicador, en términos de porcentajes, de la distancia semántica que existe entre las diferentes palabras. 
El instrumento de la Técnica de Redes Semánticas está compuesto por tres partes: la primera pedir a los participantes que definieran la palabra estímulo, que en este caso fue la palabra investigación, y el estímulo distractor, en este caso, fue realidad social; la segunda parte corresponde a la respectiva jerarquización que se asigna a las palabras asociadas a cada estímulo; y la tercera buscó obtener datos sociodemográficos, como edad, género, ocupación y ciudad de origen.

\section{Muestreo e instrumentos}

Para esta investigación se contó con la participación de 46 sujetos: 25 de género femenino y 21 de género masculino; 8 con edades entre 24 y 30 años, 21 entre 31 y 34 años y 17 mayores de 34 años. Los participantes fueron elegidos a través de un muestreo no probabilístico intencional, según disponibilidad de tiempo y acceso. Este tipo de muestreo da una base de análisis pertinente para los estudios de tipo exploratorio, bajo la premisa de la intencionalidad del muestreo hacia muestras representativas.

Se declara que en esta investigación los encuestados participaron con pleno conocimiento y consentimiento dado por escrito, siguiendo con las normas éticas de la APA.

\section{Procedimiento}

En primer lugar se eligió la palabra estímulo por evaluar (investigación), y la palabra estímulo distractor (realidad social); luego, se elaboró el instrumento para la recolección de información, o ficha sociodemográfica; posteriormente, se recogió la información en el instrumento diseñado, según las indicaciones de Valdez (2004), bajo los siguientes pasos:

1. Se pidió a los participante que definieran con sustantivos, adjetivos y otras palabras que considera se encuentran relacionadas con el estímulo (investigación) y estímulo distractor (realidad social).

2. Se solicitó jerarquizar las definiciones propuestas, teniendo en cuenta la relación o importan- cia, asignando el número 1 a la palabra más cercana al estímulo, 2 a la que sigue y así sucesivamente, hasta asignar a todas las palabras un número (de uno a diez).

3. Posteriormente se realizó el análisis de la información, con el fin de obtener del valor J, el valor M, el conjunto SAM y, por otro lado, el valor FMG.

4. Se complementó el análisis haciendo uso de la prueba Chi-cuadrado (x2), para contrastar el tamaño de red según las variables sexo y edad de la ficha socio-demográfica, con el fin de determinar si existen diferencias significativas.

\section{Resultados}

Con análisis estadísticos se determinaron los cuatro principales valores de la técnica de Redes Semánticas Naturales: Valor J, Valor M, Grupo SAM y Valor FMG.

- Valor J: los 46 encuestados registraron un total de 220 palabras diferentes asociadas a la palabra estímulo -Investigación- ( $\mathrm{J}=220$ ).

- Valor M: entre las palabras más frecuentes registradas por los 46 encuestados se encuentran: Indagación (con un valor $M$ correspondiente al $32.6 \%$ ), Búsqueda (26.1\%), Problema (26.1\%), Conocimiento (23.9\%), Metodología (23.9\%), Teoría (21.7\%), Preguntas (19.5\%), Objetivos (15.2\%), Construcción (13\%) y Escritura (10.9).

El grupo SAM corresponde a las palabras de mayor peso semántico, que es la relación existente entre la frecuencia (fi) de aparición de cada palabra y la jerarquía otorgada (pi). La jerarquía de cada palabra asociada al término Investigación es asignada por el encuestado al dar una valoración de 1 a 10 a las palabras reportadas, $x_{1}, x_{2}, \ldots, x_{10}$, según el nivel de importancia (1: baja importancia, 10: alta importancia).

El peso semántico de la palabra xi, denotado por $P_{x i}$, se obtiene a partir de la siguiente expresión:

$$
P_{x i}=\sum_{i=1}^{10} p_{i} \cdot f_{i}
$$

Siendo, 
$\rho i$ : Valor jerárquico reportado por cada encuestado a la palabra xi. Ejemplo: Indagación.

$f i$ : Frecuencia. Número de encuestados que reportaron la palabra xi. Ejemplo: Indagación.

P $\chi i$, Representa el peso semántico de la palabra xi, el cual, según Valdez (2005), está definido como la relación que hay entre la frecuencia de aparición y la jerarquía asignada por los participantes a cada una de las palabras definidoras.

Por ejemplo, sea x1 la palabra indagación (asociada al término Investigación).

$$
\begin{gathered}
P_{x 1}=\sum_{i=1}^{10} p_{i} \cdot f_{i} \\
P_{\text {Indagación }}=\sum_{i=1}^{10} p_{i} \cdot f_{i}= \\
\left(1^{*} 2\right)+\left(2^{*} 1\right)+\left(3^{*} 0\right)+\left(4^{*} 0\right)+\left(5^{*} 1\right)+\left(6^{*} 1\right)+\left(7^{*} 1\right)+
\end{gathered}
$$$$
(8 * 1)+(9 * 4)+(10 * 4)=106
$$

De manera que el peso semántico de la palabra indagación es de 106, siendo la palabra de mayor relación al término Investigación.

Las 15 palabras que reportaron mayores pesos semánticos constituyen el grupo SAM; estas son: Indagación, Problema, Búsqueda, Conocimiento,
Preguntas, Teorías, Metodologías, Construcción, Objetivos, Curiosidad, Innovación, Realidad, Método, Aprendizaje, Análisis y Disciplina.

Tabla 1.

Grupo SAM.

\begin{tabular}{lc}
\hline Palabras definidoras & $\begin{array}{c}\text { Peso semántico } \\
\text { (Px) }\end{array}$ \\
\hline Indagación & 106 \\
\hline Problema & 101 \\
\hline Búsqueda & 82 \\
\hline Conocimiento & 74 \\
\hline Preguntas & 70 \\
\hline Teoría & 63 \\
\hline Metodología & 57 \\
\hline Construcción & 44 \\
\hline Objetivos & 44 \\
\hline Curiosidad & 41 \\
\hline Innovación & 39 \\
\hline Realidad & 37 \\
\hline Método & 34 \\
\hline Aprendizaje & 34 \\
\hline Análisis & 33 \\
\hline Disciplina & 33 \\
\hline
\end{tabular}

Fuente. elaboración propia.

Figura 1.

Valor M: palabras más frecuentes asociadas al término investigación (\% de encuestados).

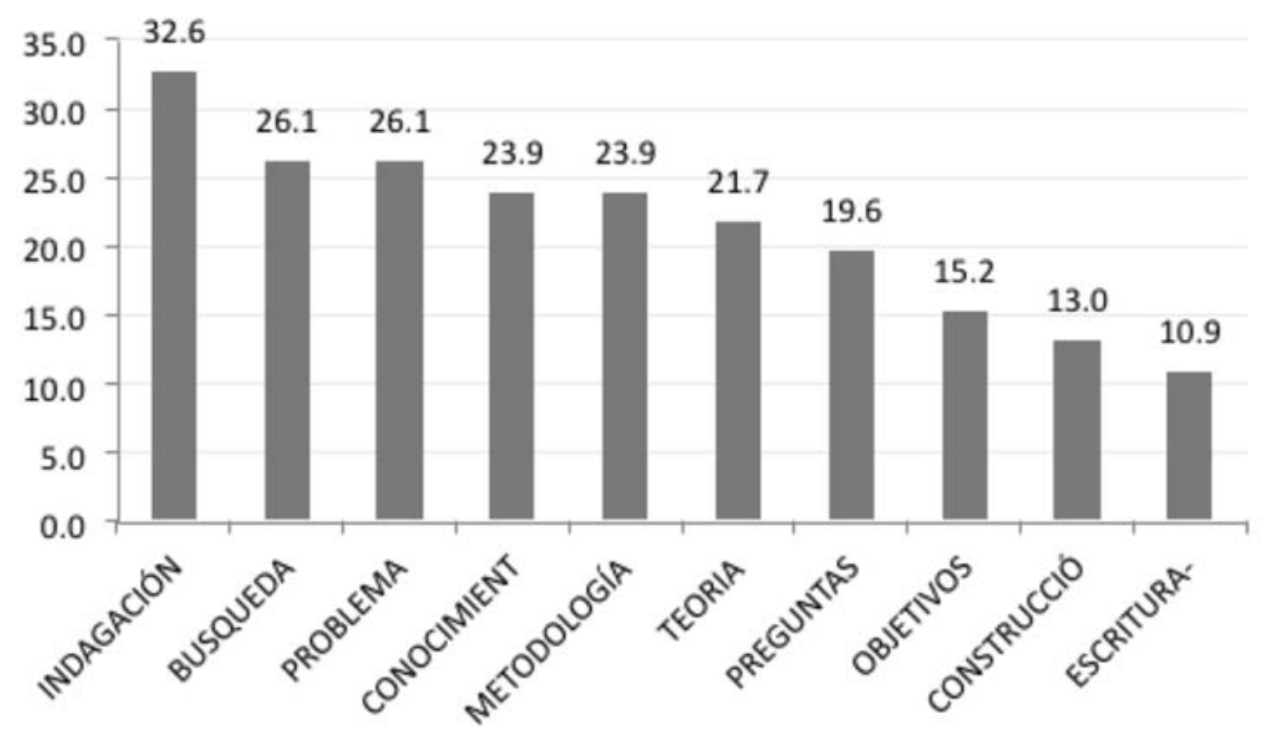

Fuente. Elaboración propia. 
El valor FMG es un indicador en términos de porcentaje de la distancia semántica que hay entre las diferentes palabras definidoras que conforman el conjunto SAM. La palabra definidora con mayor peso semántico para el estímulo Investigar, es Indagación, con un peso semántico de 106, al cual se le asigna el valor porcentual $100 \%$ (por ser el máximo peso); luego, a través de una regla de tres simple, se obtiene la distancia semántica de esta palabra, Indagación, con las otras palabras que le siguen. Así, por ejemplo, las palabras Problema y Búsqueda, que tienen un peso semántico de 101 y 82, respectivamente, determinan una distancia semántica de:

\begin{tabular}{ll} 
Peso semántico & $\%$ \\
PxMayor & 100 \\
\hline Pxi & $?$ \\
& $?=\frac{P_{x i} * 100}{P_{x \text { Mayor }}}$
\end{tabular}

Problema: $101 \times 100 / 106=95.28 \%$

Búsqueda: $82 \times 100 / 106=77.36 \%$

En la tabla 2 se indican las distancias semánticas cuantitativas (FMG) para las palabras más frecuentes asociadas al término Investigación.

Tabla 2.

Distancia semántica.

\begin{tabular}{lll}
\hline Grupo SAM & Px & FMG (\%) \\
\hline Indagación & 106 & $100 \%$ \\
\hline Problema & 101 & 95,28 \\
\hline Búsqueda & 82 & 77,36 \\
\hline Conocimiento & 74 & 69,81 \\
\hline Preguntas & 70 & 66,04 \\
\hline Teoría & 63 & 59,43 \\
\hline Metodología & 57 & 53,77 \\
\hline Objetivos & 44 & 41,51 \\
\hline Construcción & 44 & 41,51 \\
\hline Curiosidad & 41 & 38,68 \\
\hline Innovación & 39 & 36,79 \\
\hline Realidad & 37 & 34,91 \\
\hline Método & 34 & 32,08 \\
\hline Aprendizaje & 34 & 32,08 \\
\hline Análisis & 33 & 31,13 \\
\hline Disciplina & 33 & 31,13 \\
\hline
\end{tabular}

Fuente. Elaboración propia.
Se observa en la figura 2 una amplia diferencia en la importancia del peso semántico de las palabras definidoras que usan los encuestados; las seis primeras concentran, aproximadamente, el $59.43 \%$ de las palabras utilizadas para describir el término Investigación.

En relación con la última palabra del grupo SAM, que corresponde a Disciplina, representa el $31.13 \%$ de las palabras asociadas al significado psicológico del término Investigar.

Al comparar las palabras que reportan mayor frecuencia (ver figura 1), con las que presentan mayor jerarquía (peso semántico, ver tabla 1), se observa que en su mayoría son las mismas y que tienden a estar ubicadas en el mismo orden. La correlación existente entre estos dos ordenamientos es alta $(R=0.924)$.

\section{Análisis del peso promedio de algunas palabras del grupo SAM}

Si bien algunas palabras tuvieron un peso semántico alto, el número de personas que las reportan no es necesariamente alto; aspecto que se visualiza al calcular el peso promedio de importancia de cada palabra.

El peso promedio de importancia de cada palabra (x), según el número de encuestados, se obtiene a partir de la expresión:

Siendo,

$$
\bar{P}_{x}=\frac{P x}{n_{x}}=\frac{\sum_{i=1}^{10} f_{i} \cdot p_{i}}{n_{x}}
$$

$\eta_{\chi}$ : Número total de personas que reportaron la palabra $x$.

En la tabla 3 se presenta el peso semántico de algunas palabras, el número de personas que las reportan y el peso promedio respectivo.

Se observa que si bien la palabra Indagación registra el mayor peso semántico (106) y el mayor número de personas que la reportan (15), el peso promedio asignado por estas 15 personas es de 7.1, que no es el más alto. 
Figura 2.

Distancia semántica.

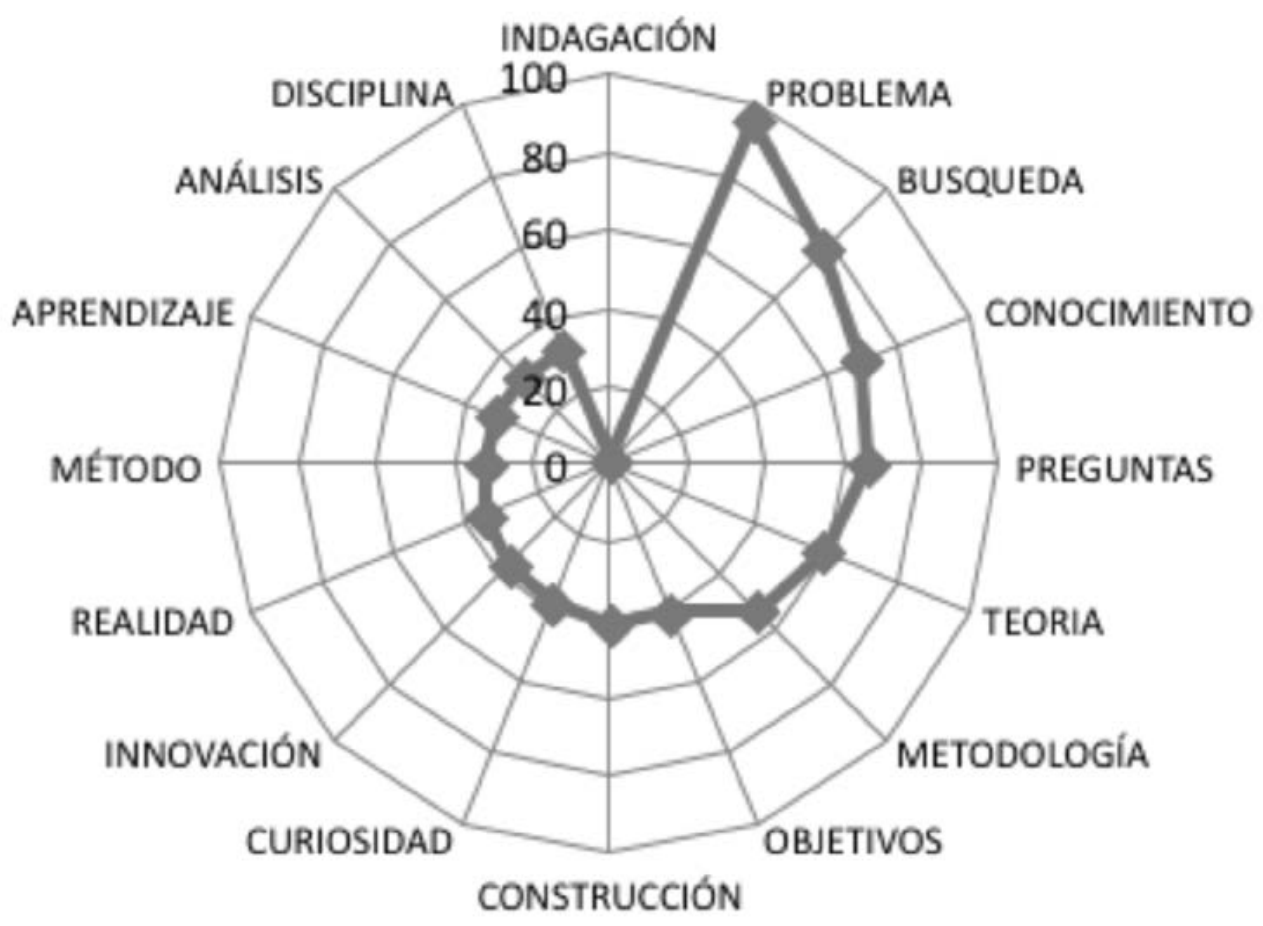

Fuente. Elaboración propia.

Tabla 3.

Peso semántico.

\begin{tabular}{lccc}
\hline Palabra & PX & Nx & Peso promedio \\
\hline Problema & 101 & 12 & 8,4 \\
\hline Pregunta & 70 & 9 & 7,8 \\
\hline Construcción & 44 & 6 & 7,3 \\
\hline Indagación & 106 & 15 & 7,1 \\
\hline Búsqueda & 82 & 12 & 6,8 \\
\hline
\end{tabular}

Fuente. Elaboración propia.

Es de anotar que dentro del valor $\mathrm{J}$ existen palabras como pasión, hallazgo, universidad y proyecto, que presentaron un alto peso promedio; sin embargo, no fueron tenidas en cuenta en razón a que el número de personas que las reportaron (fi) fue de entre 1 a 3 , valor no representativo para el análisis.
Relación entre el género y las palabras más frecuentes del valor $M$

Siendo las redes semánticas una técnica que permite obtener el significado psicológico de un término semántico (para este caso, Investigación), el cual podría tener un componente experiencial, cobra importancia analizar la relación existente entre las palabras que han obtenido mayor frecuencia de presentación (valor $M$ ) y algunas variables sociodemográficas de los participantes (género y edad).

A continuación se presenta un análisis a través de la prueba estadística Chi-cuadrado, para determinar si existe relación de dependencia entre la variable género y la frecuencia de registro de los términos asociados al concepto Investigar. En la tabla de contingencia (tabla 3 ) se relacionan los 8 
términos de mayor frecuencia (Indagación, Objetivos, Preguntas, Teoría, Búsqueda, Metodología, Conocimiento y Problema), asociados al género del participante. Se reportaron 87 registros, de los cuales 50 fueron dados por el género femenino, y 37 por el género masculino.

Tabla 4.

Relación entre género y valor $M$.

\begin{tabular}{|c|c|c|c|c|c|c|c|c|c|}
\hline 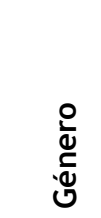 & 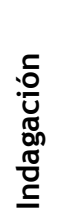 & 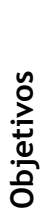 & 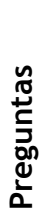 & 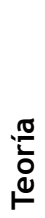 & 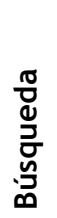 & 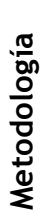 & 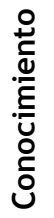 & $\begin{array}{l}\stackrel{0}{\varepsilon} \\
\frac{0}{0} \\
\frac{0}{0} \\
\frac{0}{2}\end{array}$ & $\begin{array}{l}\sqrt[\pi]{\mathbb{0}} \\
\stackrel{\circ}{\circ}\end{array}$ \\
\hline$F$ & 7 & 4 & 7 & 5 & 8 & 6 & 5 & 8 & 50 \\
\hline$M$ & 8 & 3 & 2 & 5 & 4 & 5 & 6 & 4 & 37 \\
\hline TOTAL & 15 & 7 & 9 & 10 & 12 & 11 & 11 & 12 & 87 \\
\hline
\end{tabular}

Fuente. Elaboración propia. Datos.

$$
\chi^{2}=\sum_{i=1}^{f} \sum_{j=1}^{c} \frac{\left(A_{i j}-E_{i j}\right)^{2}}{E_{i j}}
$$

Aij = frecuencia observada en la i-ésima fila, $j$ ésima columna

Eij = frecuencia esperada en la i-ésima fila, j-ésima columna,

Eij $=\left(\right.$ Total de registros de la fila $i^{*}$ total de registros de la columna j)/(total de registros)

$r=$ número de filas (Fila: Género. $r=2$ )

$c=$ número de columnas (columna: términos de mayor frecuencia, $\mathrm{c}=8$ )

El estadístico de prueba $\chi^{2}=0,78182864$ es comparado con el percentil de la distribución $\chi^{2}=$ con $(2-1)^{*}(8-1)=7$ grado de libertad, a un nivel de significancia del $5 \%$, resultando un valor crítico de $\chi_{(7)}^{2}=14.07$. Por lo tanto, como el valor del estadístico no es superior al valor crítico, se concluye que no existe relación entre el género del encuestado y los registros más frecuentes de las palabras asociadas al concepto Investigación.

\section{Relación entre edad y palabras más frecuentes del valor $M$}

Considerando que el significado psicológico puede estar relacionado con las experiencias de vida, y para este caso con la experiencia del proceso de investigar, se quiere determinar si existe relación entre la variable sociodemográfica edad y los registros más frecuentes del grupo $M$. Para lo anterior, se realizó una prueba Chi-Cuadrado $\left(\chi^{2}\right)$. En la tabla 4 se muestran los datos correspondientes a la tabla de contingencia.

Tabla 5.

Relación entre edad y valor M.

\begin{tabular}{|c|c|c|c|c|c|c|c|c|c|}
\hline 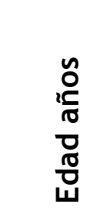 & 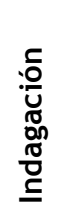 & 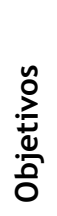 & 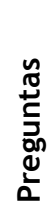 & 苾 & 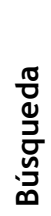 & 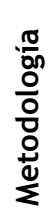 & 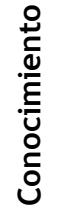 & 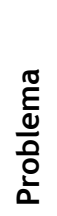 & $\underset{1}{\frac{1}{6}}$ \\
\hline $\begin{array}{l}\text { Menor } \\
\text { de } 24\end{array}$ & 0 & 0 & 1 & 0 & 1 & 0 & 1 & 0 & 3 \\
\hline $4-30$ & 4 & 0 & 1 & 0 & 0 & 2 & 0 & 1 & 8 \\
\hline $31-44$ & 5 & 3 & 3 & 6 & 5 & 4 & 3 & 5 & 34 \\
\hline $\begin{array}{l}\text { Mayor } \\
\text { de } 34\end{array}$ & 6 & 3 & 4 & 3 & 6 & 4 & 7 & 6 & 39 \\
\hline Total & 15 & 6 & 9 & 9 & 12 & 10 & 11 & 12 & 84 \\
\hline
\end{tabular}

Fuente. Elaboración propia.

El estadístico de prueba $\chi^{2}=0,9708$ es comparado con el percentil de la distribución $\chi^{2}$ con $(4-1)^{*}(8-1)$ $=21$ grado de libertad, a un nivel de significancia del $5 \%$, resultando un valor crítico de $\chi_{(21)}^{2}=$ 32.67. Por lo tanto, como el valor del estadístico no es superior al valor crítico, se concluye que no existe relación entre la edad del encuestado y los registros más frecuentes asociados al concepto investigación. Es decir, la edad de los participantes no presentó relación significativa con las palabras asociadas al concepto investigación.

\section{Discusión}

A partir de los resultados obtenidos, se evidencia que los encuestados identifican un número amplio de conceptos asociados a la palabra Investigar 
(reportando un valor $\mathrm{J}$ de 220 palabras diferentes), lo que sugiere una diversidad del significado de lo que implica investigar, así como lo refieren Rips, Shoben y Smith (1973). Asimismo, este valor J permite conocer la riqueza lexicográfica del grupo de participantes, lo que deja ver la amplitud de significados que tiene el concepto. Este valor es importante porque indica qué tanto asociaron los docentes-investigadores sobre la palabra estímulo (Investigación). La amplitud del valor J sugiere la procedencia y formación académica de los participantes, dejando un interrogante frente a otros posibles grupos participantes con procedencia y formación diferentes.

Dentro de las palabras más frecuentes se encuentran Indagación y Búsqueda, con porcentajes de frecuencia de aparición de $32.6 \%$ y $26.1 \%$, respectivamente; si se consideran estas palabras como sinónimas, podría interpretarse que el 58.7 $\%$ de los encuestados consideran, en principio, el carácter etimológico (Investigare-ir en vestigium) de la palabra Investigación. Siendo quizás lo anterior una explicación de aquellos procesos investigativos que en gran medida quedan en la etapa de búsqueda y exploración. Este valor $M$ resultante de la técnica de Redes Semánticas $\mathrm{Na}$ turales sobre la misma palabra estímulo sugiere una transformación de la escala de los significados otorgados, según la preferencia y vinculación afectiva con la actividad investigativa.

Los valores generales del conjunto SAM encontrados para el estímulo “investigación”, está asociado principalmente a los términos Indagación, Problema y Búsqueda, con pesos semánticos de 106, 101 y 82 , respectivamente; siendo estas las tres palabras de mayor peso semántico. En contraste, se encuentran las palabras Método y Aprendizaje, con peso semántico de 34, y las palabras Análisis y Disciplina, con peso semántico de 33. Lo anterior sugiere la preferencia del uso de metodologías cualitativas en el proceso de investigar.
Desde el valor FMG, la distancia semántica entre los términos Indagación y Método es de aproximadamente el $70 \%$. Adicionalmente, se observa que aquellos términos que implican mayor formalidad y rigor, como Método y Disciplina (conceptos que se encuentran relacionados históricamente con el "método científico" desde una perspectiva positivista), obtuvieron pesos semánticos bajos; lo anterior sigue sugiriendo una posible tendencia del método investigativo de tipo cualitativo, propio de la formación en Ciencias Sociales, que corresponde a la de la mayoría de los asistentes al Evento Vendimia 2012.

En este estudio no se realizaron categorías para el análisis de los términos asociados a la palabra estímulo, como lo hacen estudios como los de García y Jiménez (1996), lo que posibilitó comprender el significado psicológico y su proceso cognitivo relacionado con la experiencia de los participantes, permitiendo captar la propia lógica de la característica del grupo, que en este caso está integrado, en su mayoría, por docentes-investigadores de las áreas de las ciencias sociales, con experiencias previas en investigación desde diversas metodologías, entre ellas, la etnografía, el estudio de caso y las historias de vida, que minimizan el uso de datos cuantitativos.

A través del análisis Chi-Cuadrado se concluye que no existe relación significativa entre el significado psicológico del concepto Investigación y variables sociodemográficas como género y edad, lo que puede sugerir que existen la presencia de otras variables que influyen en la construcción del concepto como la profesión o área de conocimiento. Los resultados reafirmaron el adecuado uso de la técnica de redes semánticas como instrumento que permite conocer y recoger el significado psicológico de la palabra estímulo con el concepto Investigar. 


\section{Referencias}

Ávila, H. (2006). Introducción a la metodología de la investigación. México: Eumed.net. Recuperado el 8 agosto de 2014 de http://books. google.es $/$ books?id=r93TK4EykfUC\&dq=\%C3\% 81vila, $+\mathrm{H} .+(2006) .+$ Introducci\%C3\%B3n+a+la+ metodolog\%C3\%ADa+de+la+investigaci\%C3\%B $3 n \& l r=\& h l=e s \&$ source=gbs_navlinks_s

Aceituno, H., Lanza, J., Lanz, J. y Lander, G. (1998). Interés, conocimientos, recursos y limitaciones de los estudiantes de medicina en la investigación científica. Gac Méd Carácas, 106(1), 40-46.

Ausubel, D., Novak, J. y Hanesian, H. (1983). Psicología educativa: Un punto de vista cognoscitivo (2da. ed.). México: Trillas.

Ander-Egg, E. (1992). Técnicas de investigación social. México. El Ateneo.

Best, J. W. (1982). Cómo investigar en educación. Capítulo I: significado de investigación. España: Morata.

Cívicos, A. y Hernández, M. (2007). Algunas reflexiones y aportaciones en torno a los enfoques teóricos y prácticos de la investigación en trabajo social. Revista Acciones e Investigaciones Sociales, 23, 25-55.

Colom, R. y Flores-Mendoza, C. (2001). Inteligencia y memoria de trabajo: la relación entre Factor G, Complejidad Cognitiva y Capacidad de Procesamiento. Psicología: Teoría e Pesquisa, 17(1), 37-47.

Cordero, Z. R. (2009). La investigación aplicada: una forma de conocer las realidades con evidencia científica. Revista Educación, 33(1) 155-165.

Figueroa, J. (1980). Orígenes de las redes semánticas naturales. Facultad de Psicología, trabajo inédito. México: UNAM.

García, C. B. y Jiménez, V. S. (1996). Redes semánticas de los conceptos de presión y flotación en estudiantes de bachillerato. Revista
Mexicana de Investigación Educativa, 1(2), 343-361.

García, F. y Doménech, F. (1997). Motivación, aprendizaje y rendimiento escolar. Revista Electrónica de Motivación y Emoción, 1(0). Recuperado de http://reme.uji.es/articulos/ pa0001/texto.html

Hergenhahn, B. (2008). Introducción a la historia de la psicología. Primera edición, segunda reimpresión. España.

Leahey, T. H. (1998). Historia de la psicología: principales corrientes en el pensamiento psicológico. Madrid: Prentice-Hall Iberia, cuarta edición.

Martínez, M. (2004). Los grupos focales de discusión como método de investigación. Heterotopía, 26, 59-72.

Moscovici, S. (1984). The phenomenon of social representations. R. M. Farr and S. Moscovici (Ed.). Social representations, 3-69. Cambridge: University Press.

Osgood, C., Suci, G. y Tannenbaum, P. (1957). The measurement of meaning. University of Illinois Press.

Padrón, J. (2006). Investigar, reflexionar y actuar en la práctica docente. Recuperado de http:// padron.entretemas.com/InvAplicada/index. htm

Ortiz, L. (2012). Curso investigación cualitativa. Bogotá: Universidad Nacional Abierta y a Distancia.

Rips, L., Shoben, E. y Smith, E. (1973). Distancia Semántica y la verificación de las relaciones semánticas. Diario de aprendizaje verbal y conducta verbal, 12(1), 1-20. Recuperado el 8 de agosto de 2014 de http:// www.sciencedirect.com/science/article/pii/ S0022537173800568

Salas-Menotti, Irene. (2008). Significado psicológico de la violencia y la agresión en una 
muestra urbana colombiana. Diversitas, 4(2), 331-343. Recuperado el 22 de septiembre 2015 de http://pepsic.bvsalud.org/ scielo.php?script=sci_arttext\&pid=S1794$99982008000200010 \& \operatorname{lng}=p t \& \operatorname{lng}=e s$

Tapia, J., Juan, A. y Huerta y Ruiz, A. (2010). On the nature of motivational orientations: Implications of assessed goals and gender differences for motivational goal Theory. The Spanish Journal of Psychology, 13, 232-243.

Valdez, L. (2004). Las redes semánticas naturales, uso y aplicaciones en psicología social. México: Universidad Autónoma del estado de México.
Vargas, S. (2009). La investigación aplicada: una forma de conocer las realidades con evidencia científica. Revista Educación, 33, 155-165.

Vera, J., Pimentel, C. y Batista, F. (2005). Redes semánticas: aspectos teóricos, técnicos, metodológicos y analíticos. Revista Ximhai, 1(3), 439-451.

Vivas, J., Comesaña, A. y Vivas, L. (2007). Evaluación de las redes semánticas de conceptos académicos en estudiantes universitarios. Psico-USF, 12(1), 111-119. 